\title{
Vorbeugender Hochwasserschutz in Flusseinzugsgebieten
}

\author{
Konsequenzen für die Raumplanung
}

\section{Preventive Flood Protection in River Catchment Areas}

Consequences for spatial planning

\section{Kurzfassung}

Dezentrale Hochwasserschutzkonzepte, bezogen auf das gesamte Einzugsgebiet kleinerer und mittlerer Flüsse, existieren bisher noch nicht. Der für das Gewässereinzugsgebiet der Wesenitz modellhaft entwickelte "Informelle Plan zum vorbeugenden Hochwasserschutz" wird als ein verallgemeinerungsfähiges Strategiekonzept der Raumplanung zur Verbesserung der Gebiets- und Gewässerretention vorgestellt. Mit dieser Strategieentwicklung sollen die Defizite in der räumlichen Planung auf dem Gebiet des vorbeugenden Hochwasserschutzes abgebaut werden. Der am Gewässereinzugsgebiet ausgerichtete Plan überwindet administrative Grenzen und fördert die interkommunale Zusammenarbeit auf dem Gebiet des Hochwasserschutzes. Für das Einzugsgebiet werden aus der Synopse wichtiger Einflussfaktoren auf den Oberflächenwasserabfluss Konfliktbereiche für die Hochwasserentstehung ermittelt. Sie bilden für die Kommunen und die Fachplanungen die Grundlage für die lokale Verteilung und Prioritätensetzung dezentraler Hochwasserschutzmaßnahmen. Die unterschiedlichen dezentralen Hochwasserschutzmaßnahmen werden in einem informellen Plan durch spezifische Planzeichen symbolisiert.

\section{Abstract}

Decentral flooding control concepts for whole drainage areas of small and medium-sized rivers do not exist so far. The "Informal Plan for preventive flood control" modelled for the drainage area of the river Wesenitz is presented as a generalisable regional-planning strategy concept devoted to improving the retention of land and water. This strategy advance will serve to remove shortcomings in spatial planning in the sphere of preventive flood control. The plan for the drainage area overcomes administrative hurdles and promotes co-operation between local authorities in the field of flood control. Using a synopsis of key factors influencing surface-water run-offs in the drainage area, conflict types with a bearing on how flooding arises are identified. These provide local authorities and sectoral planners with a means of specifying local flooding measures and of establishing priorities in respect thereof. Different local flooding measures are represented in the Informal Plan by means of special planning symbols. 


\section{Vorbemerkungen}

Vorbeugender Hochwasserschutz ist nicht ausschließlich eine wasserwirtschaftliche Aufgabe, auch die Raumordnung muss einen wesentlichen Beitrag zum Hochwassermanagement leisten. Die für Raumordnung in Deutschland zuständigen Minister fordern deshalb die Einrichtungen der Raumordnung auf, ihre Instrumente zum Erhalt und zur Wiederherstellung von Retentionsräumen einzusetzen, auf eine Verbesserung des Wasserrückhalts im Einzugsgebiet und auf eine vorsorgende Risikobeachtung in überflutungsgefährdeten Räumen hinzuwirken (Handlungsempfehlungen der MKRO 2000). In diesem Zusammenhang fordern die Minister auch, dass die geeigneten Instrumente und Maßnahmen handlungsbezogen konkretisiert werden und dass sich das raumordnerische Flächenmanagement auf die Flächen des gesamten Gewässereinzugsgebietes beziehen muss. Um dem gerecht zu werden, hat die Raumordnung Strategien zu entwickeln, die eine grenz- und sektorübergreifende Kooperation zwischen Kommunen, Regionen, Ländern und Staaten einerseits und den Fachplanungen andererseits, insbesondere mit der Wasserwirtschaft, dem Natur- und Landschaftsschutz, der Land- und Forstwirtschaft und dem Städtebau, ermöglichen.

\section{Ziele des vorbeugenden Hochwasserschutzes}

Das raumordnerische Flächenmanagement hat bezüglich des vorbeugenden Hochwasserschutzes darauf Einfluss zu nehmen, dass die Niederschläge im gesamten Flusseinzugsgebiet so lange wie möglich auf der Fläche zurückgehalten und so verzögert wie möglich dem Vorfluter zugeführt werden und dass der Niederschlagsabfluss sich im Gewässerbett bei einer möglichst niedrigen Abflussgeschwindigkeit gefahrlos ausbreiten kann. Das heißt, neben der Sicherung von Überschwemmungsgebieten ist vor allem das natürliche Retentionsvermögen auf der Landoberfläche und im Gewässer zu erhöhen, um den Oberflächenwasserabfluss zu dämpfen. Dafür gibt es eine Reihe unterschiedlicher dezentraler Maßnahmen, die in der Regel auch der Praxis bekannt sind und sich im Vergleich zum technischen Hochwasserschutz wesentlich investitionsgünstiger und vor allem umweltverträglicher in Verantwortung der Kommunen umsetzen lassen. Zwar zeigen solche dezentrale Maßnahmen einzeln oft nur eine geringe Wirkung, sie führen aber in der Addition zu einem erheblichen Summeneffekt und leisten in ihrer Gesamtheit einen beträchtlichen Beitrag zur Verbesserung der Gebiets- und Gewässerretention. Da es sich aber hierbei um Maßnahmen handelt, für die unterschiedliche Fachplanungen zuständig sind, wurden sie bisher nur selten als ein Gesamtpaket umgesetzt. Hinzu kam, dass der Hochwasserschutz vorrangig eine Domäne der Wasserwirtschaft war und er deshalb in den Raumordnungsplänen nur selten thematisiert wurde (Kistenmacher 1996, 1.6). Aber auch in den raumrelevanten Fachplänen ging es bisher vordergründig meist nur um fachspezifische Fragestellungen des Naturschutzes, der Gewässerreinhaltung, der landund forstwirtschaftlichen Entwicklung im Zusammenhang mit ökonomischen und ökologischen Zielen, aber in den seltensten Fällen darum, Niederschlagswasser durch Vorgaben zur Landnutzung, Bodenbewirtschaftung, Bodenversiegelung oder Regen- und Abwasserbewirtschaftung in Siedlungsbereichen am schnellen Abfluss zu hindern bzw. zurückzuhalten. Es erscheint deshalb zwingend, für den vorbeugenden Hochwasserschutz ein Planungsinstrument zu entwickeln, das flusseinzugsgebietsbezogen alle dezentralen Maßnahmen bündelt und die Handlungsoptionen den Fachplanungen und Kommunen zur Umsetzung vorgibt.

\section{Hochwasserschutz auf kommunaler Ebene}

Um aus einer komplexen Sicht Einfluss auf eine Umsetzung der dezentralen Maßnahmen zum vorbeugenden Hochwasserschutz auf kommunaler Ebene zu nehmen, wurde im Dezember 2000 vom Institut für ökologische Raumentwicklung e. V. in Kooperation mit dem Umweltbundesamt Berlin ein internationaler Workshop „Vorbeugender Hochwasserschutz auf kommunaler Ebene" in Dresden durchgeführt. Auf diesem Workshop diskutierten Vertreter des Bundesumweltministeriums, Umweltbundesamts und der kommunalen Spitzenverbände mit den Vertretern aus Forschung, Planung und Kommunen. Hauptanliegen der Veranstaltung war es, die Gemeinden dafür zu sensibilisieren, dass Hochwasserschutz nicht nur in den unmittelbaren Gefahrenbereichen am Hauptgewässer, sondern auf dem gesamten Gemeindegebiet zu erfolgen hat (Greiving 1999, S. 25). Auf dem Workshop wurde einerseits herausgearbeitet, dass die Kommunen eine zentrale Rolle bei der Umsetzung der vorbeugenden Hochwasserschutzmaßnahmen übernehmen müssen. Andererseits wurde aber auch deutlich, dass die Raumplanung die interkommunale Zusammenarbeit in den Flusseinzugsgebieten stärker fördern muss. Insbesondere die Regionalplanung muss in ihrer Verantwortung eine Moderatorenfunktion übernehmen und in dieser Rolle in den Gemeinden sowie bei den einschlägigen Behörden das Bewusstsein dafür entwickeln, dass alle in einem Flusseinzugsgebiet liegenden Gemeinden unabhängig von der unterschiedlichen Hochwasserbetroffenheit eine Solidargemeinschaft bilden müssen. 
Auf dem Workshop wurde vom Institut für ökologische Raumentwicklung e. V. der an einem Modellgebiet entwickelte „Informelle Plan zum vorbeugenden Hochwasserschutz" als ein verallgemeinerungsfähiges Strategiekonzept der Raumplanung zur Verbesserung der Gebiets- und Gewässerretention zur Diskussion gestellt. Er soll durch unterschiedliche Publikationen und Verbreitung im Internet einer noch größeren Öffentlichkeit vorgestellt werden, um den Planungsprozess zum vorbeugenden Hochwasserschutz zu beschleunigen und in den Kommunen die Umsetzung dezentraler Hochwasserschutzmaßnahmen voranzubringen.

\section{Der informelle Plan zum vorbeugenden Hochwasserschutz als Strategiekonzept}

\subsection{Zielstellung}

Für Flusseinzugsgebiete sind nach den Entschließungen der Ministerkonferenz für Raumordnung (MKRO 2000) gesamträumliche Konzepte und Handlungsprogramme zum vorbeugenden Hochwasserschutz zu entwickeln. Das vorgestellte Konzept verfolgt auf der Basis einer flusseinzugsgebietsbezogenen Analyse die Ermittlung der Konfliktbereiche, die zum erhöhten Oberflächenwasserabfluss in der Landschaft beitragen. Diese Ermittlung soll für die Belange der Raumordnung geeignet und ausreichend sein und auf eine umfangreiche Niederschlags-Abfluss-Modellierung verzichten. Für diese Konfliktbereiche sollen, durch die Regionalplanung koordiniert, die lokal günstigsten Leitbilder zur Dämpfung des Oberflächenwasserabflusses ermittelt und den Fachplanungen sowie den Kommunen zur Umsetzung vorgegeben werden.

\subsection{Gebietsanalyse}

Für das Modellgebiet der Wesenitz, einem Nebenflusseinzugsgebiet im Bereich der Oberen Elbe, wurden zunächst alle biotischen, abiotischen und anthropogenen Einflussfaktoren, die die Hochwasserbildung begünstigen, erfasst und problembezogen bewertet. Untersuchungen wurden geführt zur Hangneigung, zur Tal- und Flussbettgeometrie, zur geologischen Struktur, zu den Bodenarten und deren Wasseraufnahmeund Wasserspeichervermögen sowie zu den Niederschlägen einschließlich ihrer Intensität, zeitlichen Verläufen und lokalen Verteilung im Einzugsgebiet, zur Landnutzung, zum Besiedlungsgrad, zur Abwasserbehandlung, Regenwasserbewirtschaftung und zum Gewässerausbau.
Im Ergebnis stellte sich die Dominanz landwirtschaftlicher Nutzungen mit meist großen ungegliederten Feldstrukturen für die Gebietsretention des Untersuchungsraumes als besonders negativ heraus. Ferner fehlen auf den überwiegend geneigten Flächen abflusshemmende Strukturelemente. Morphologisch dominieren die flach- bis lehnhängigen Geländeberejche. Der lehn- bis steilhängige Anteil, der den Niederschlagsabfluss besonders beschleunigt, nimmt über $16 \%$ der Gesamtfläche ein. Der Waldanteil ist mit einem Fünftel der Fläche sehr gering, er liegt erheblich unter dem sächsischen Landesdurchschnitt und trägt nur wenig zur Verzögerung des Niederschlagsabflusses bei. Als ungünstig für die Gebietsretention erweisen sich auch die Bodenverhältnisse. In mehr als zwei Dritteln des Gebietes liegen lehm- und lößhaltige Böden an, das restliche Drittel bilden sandige Böden. Der Siedlungs- und Verkehrsflächenanteil deckt etwa $9 \%$ des Untersuchungsgebietes ab und liegt unter dem bundesdeutschen Durchschnitt. Bezüglich der Gewässerretention begünstigen Gewässerlaufbegradigungen und Gewässerausbauten größeren Ausmaßes in den Siedlungsbereichen lokal einen schnellen Oberflächenwasserabfluss. Die Abwasserbehandlung erfolgt kommunal noch sehr unterschiedlich. In den ländlichen Kommunen herrschen dezentrale Sammelanlagen und Mischsysteme vor, in städtischen Bereichen dagegen zentrale Anlagen mit Trennsystemen. Eine Regenwasserbewirtschaftung in den Siedlungsbereichen hat sich nur in Ausnahmefällen, meist in Verbindung mit neuen Gewerbe- und Wohnsiedlungen, durchgesetzt.

\subsection{Ermittlung der Konfliktbereiche für die Hochwasserbildung}

Die Ermittlung der potenziellen Konfliktbereiche erfolgte über die synoptische Bewertung ausgewählter Einflussfaktoren. In Auswertung der Untersuchungsergebnisse kann für ein Konzept in der mittelmaßstäblichen Arbeitsebene der Regionalplanung verallgemeinert ausgesagt werden, dass in erster Linie die Einflussgrößen Hangneigung, Wasserdurchlässigkeit der Böden und Landnutzung zu analysieren sind. Diese Faktoren üben den größten Einfluss auf die Intensität des Oberflächenwasserabflusses in der Landschaft aus und bestimmen wesentlich die Handlungsoptionen für den vorbeugenden Hochwasserschutz. Die detaillierten Aussagen zur Hangneigung und zur Wasserdurchlässigkeit der Böden lieferten für den Modellraum die Daten aus den Mikrogeochoren, die flächendeckend für den Freistaat Sachsen vorliegen. Für die Landnutzung wurden die Aussagen aus den Satellitenbilddaten von CORINE Land Cover bezogen. Für die mittelmaß- 


\section{Abbildung 1}

Hangneigungsflächentypen im Einzugsgebiet der Wesenitz - Methodik der Zusammenfassung

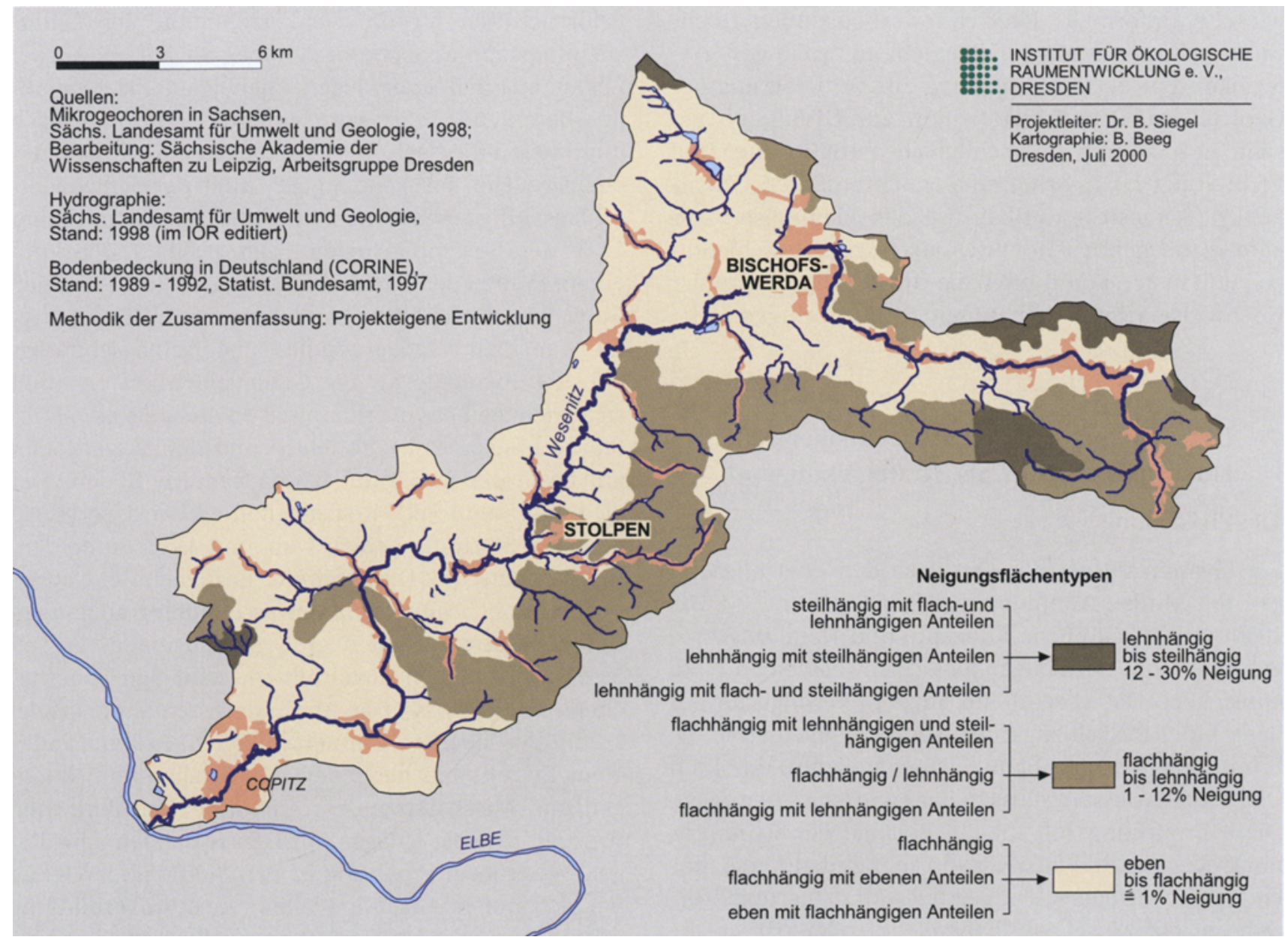

stäbliche Arbeitsebene mussten allerdings die zum Teil sehr detailliert vorliegenden Daten zusammengefasst werden. Beispielhaft wird das an der thematischen Karte zur Hangneigung (Abb. 1) demonstriert. Hier wurden die für das Einzugsgebiet aus den Mikrogeochoren gewonnenen neun Neigungsflächentypen in drei Gruppen mit den Aussagen lehnhängig bis steilhängig, flachhängig bis lehnhängig und eben bis flachhängig zusammengefasst.

Die Bewertung geht davon aus, dass der Niederschlagsabfluss mit steigender Hangneigung zunimmt und dass er auf landwirtschaftlichen Nutzflächen intensiver ist als auf Waldflächen (Pessel 1997, S. 25). Außerdem wird angenommen, dass der Niederschlagsabfluss auf bindigen Böden stärker als auf sandigen Böden ist.
Damit ergeben sich für die Intensität des Niederschlagsabflusses die vier Stufen: stark, mäßig, gering, sehr gering (Abb. 2). Diese unterschiedlichen Abflussintensitäten sind unter Verwendung der genannten Daten für das gesamte Einzugsgebiet in einer Konfliktkarte (Abb. 3) dargestellt. Aus der Intensität des Oberflächenwasserabflusses lassen sich die Prioritäten für die mittel- bis langfristige Umsetzung der Maßnahmen ableiten. Die vorgenommene Methodik zur Ermittlung der Intensität des Oberflächenwasserabflusses kann aus raumordnerischer Sicht für Planungen zum vorbeugenden Hochwasserschutz empfohlen werden. Die Ergebnisse sind zwar grob, sie liefern aber für die Ebene der Regionalplanung vor allem kurzfristig eine ausreichende Planungsschärfe. 


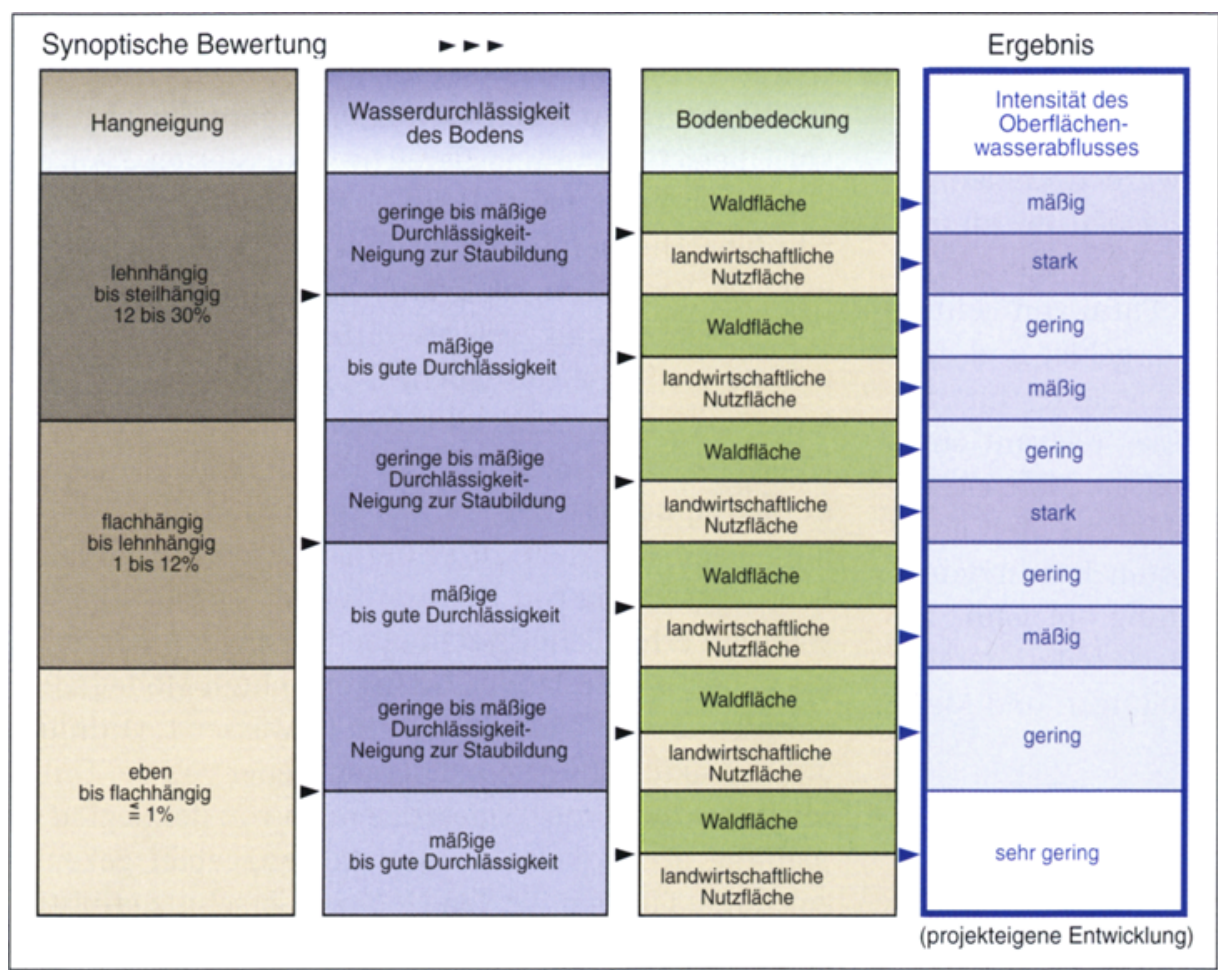

Abbildung 2

Ermittlung der Intensität

des Oberflächenwasserabflusses

\section{Abbildung 3}

Intensität des Oberflächenwasserabflusses im Einzugsgebiet der Wesenitz - Ergebnis der synoptischen Bewertung aus Hangneigung, Bodentypen und Bodenbedeckung

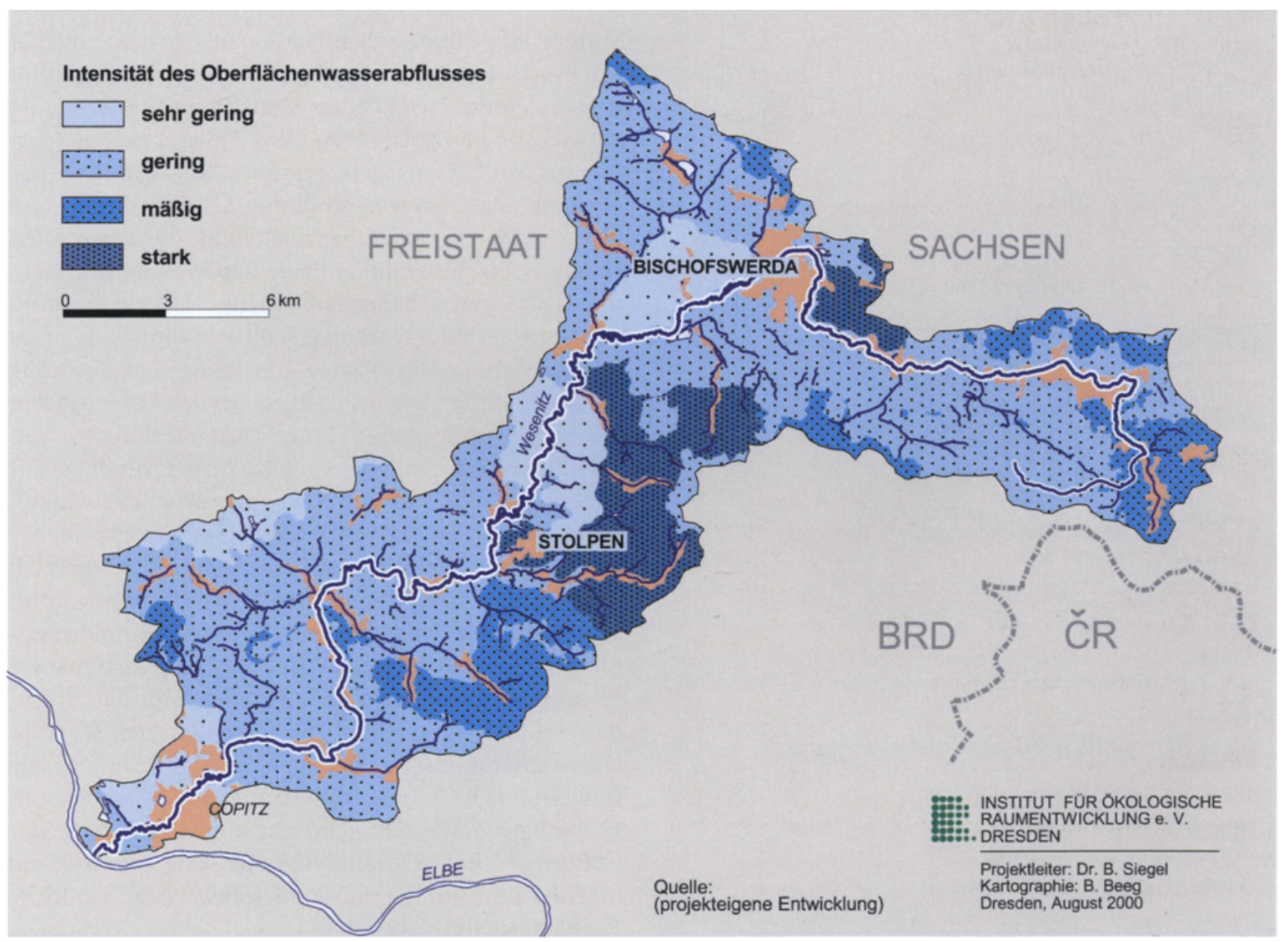




\subsection{Planzeichen für dezentrale Maßnahmen}

Um der Praxis die breite Palette der dezentralen Maßnahmen aufzuzeigen, die zur Dämpfung des Niederschlagsabflusses beitragen können, wurden 15 Planzeichen (Abb. 4), die sich an die Planzeichenverordnung anlehnen, entwickelt. Sie symbolisieren die unterschiedlichen Handlungsoptionen in Form von Leitbildern, die auf den Flächen im Einzugsgebiet und entlang aller darin liegenden Gewässerläufe für die Dämpfung des Niederschlagsabflusses relevant sind. Im unbesiedelten Bereich orientieren die Planzeichen in erster Linie auf die vielfältigen Möglichkeiten einer an den Hochwasserschutz angepassten Landnutzung und -bewirtschaftung, z.B. Aufforstung auf lehn- bis steilhängig geneigten Flächen, im besiedelten Bereich dagegen stärker auf Flächenentsiegelungen und Maß-

\section{Abbildung 4}

Planzeichen für dezentrale Hochwasserschutzmaßnahmen

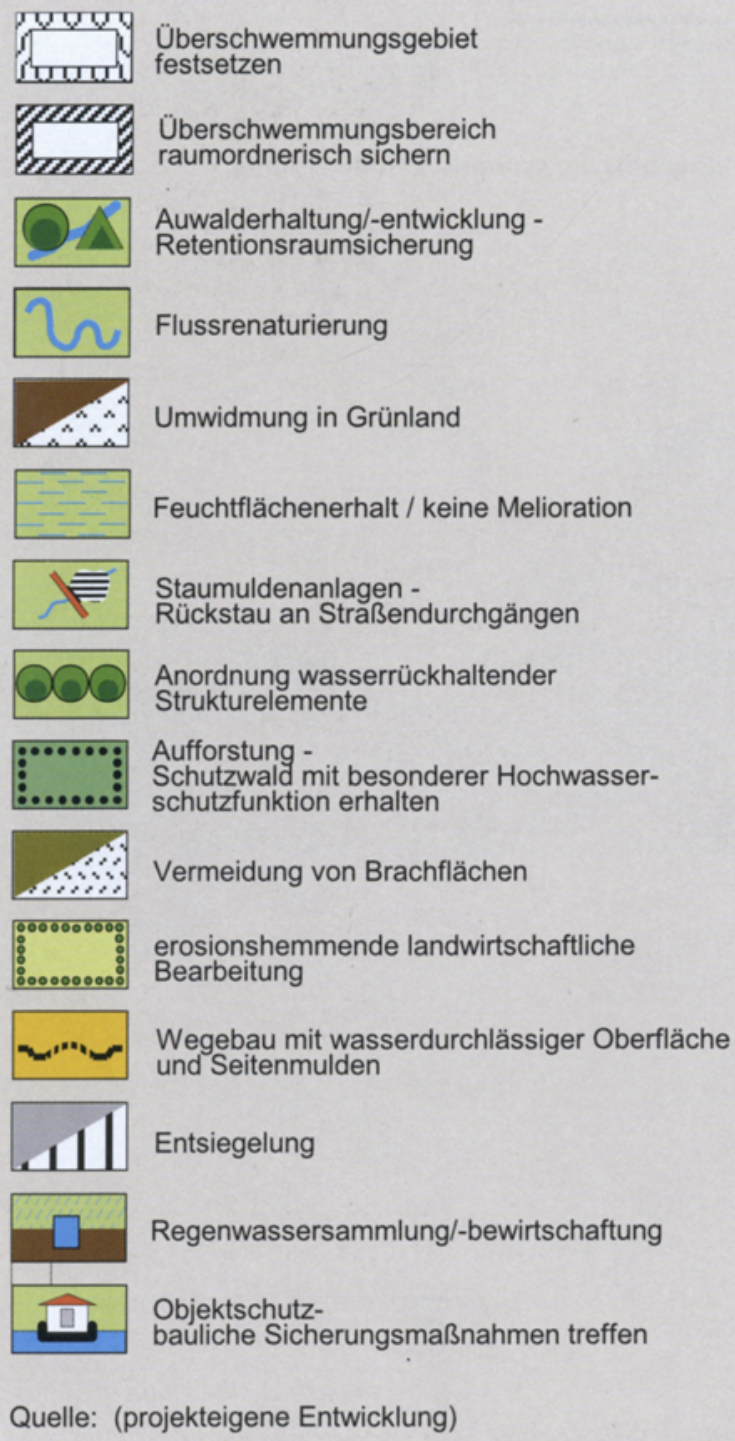

nahmen zur Regenwasserbewirtschaftung. Entlang der Gewässer verfolgt die Raumplanung neben Hinweisen zur Renaturierung von Gewässerabschnitten mit den auf Überschwemmungsbereiche fokussierten Planzeichen zwei Ziele: Erstens sollen überschwemmungsgefährdete Uferbereiche im unbebauten Bereich mit weniger Verwaltungsaufwand „raumordnerisch gesichert" werden und zwar als Vorbehalts- bzw. Vorranggebiete im Sinne des Hochwasserschutzes und zweitens soll mit dem Planzeichen „festzusetzendes Überschwemmungsgebiet" an die Unteren Wasserbehörden appelliert werden, Überschwemmungsgebiete umgehend rechtlich zu sichern. Für die Ausweisung bzw. Festsetzung von Überschwemmungsgebieten besteht ein erheblicher Nachholbedarf, insbesondere für die neuen Bundesländer. Im untersuchten Modellraum konnten für die Wesenitz als ein Gewässer 1. Ordnung aus den aktuellen Regionalplänen weder von der Unteren Wasserbehörde festgesetzte noch von der Regionalplanung als Vorbehalts- oder Vorranggebiet gekennzeichnete Flächen für den Hochwasserschutz ermittelt werden.

\subsection{Informeller Plan zum vorbeugenden Hochwasserschutz}

Mithilfe eines informellen Planes soll der vorbeugende Hochwasserschutz stärker in die Raumplanung einbezogen werden. Der für den Modellraum der Wesenitz entwickelte informelle Plan (Abb. 5) leitet sich aus den für das. Flusseinzugsgebiet ermittelten Konfliktbereichen für die Hochwasserbildung ab. Die standortgerechte Ausweisung der Planzeichen für die dezentralen Hochwasserschutzmaßnahmen hat in einem intensiven Diskurs zwischen sachkundigen lokalen Akteuren und Vertretern der einschlägigen Fachplanungen zu erfolgen. Wichtige Hinweise für die richtige Lokalisierung der Maßnahmen leiten sich auch aus den kommunalen Flächennutzungsplänen und aus Luftbilddaten von Satellitenüberfliegungen ab. Die Kartengrundlage für den informellen Plan zum vorbeugenden Hochwasserschutz bildet die TOP-Karte des Flusseinzugsgebietes. Der Maßstab sollte im mittelmaßstäblichen Bereich liegen. Aus Übersichtsgründen sollte die topographische Karte auf ausgewählte Daten der Geoinformationssysteme Hydrographie, Straßen- und Verkehrsnetz sowie Bodenbedeckung reduziert und mit den ermittelten Konfliktbereichen zur Intensität des Oberflächenwasserabflusses überlagert werden. Kommunale und regionale Grenzen sowie Landes- und Staatsgrenzen sollten dargestellt sein, um unterschiedliche Verwaltungs- und Zuständigkeitsbereiche sichtbar zu machen und um ggf. auf landesspezifische rechtliche Regelungen hinzuweisen. 


\section{Abbildung 5}

Informeller Plan zum vorbeugenden Hochwasserschutz im Einzugsgebiet der Wesenitz

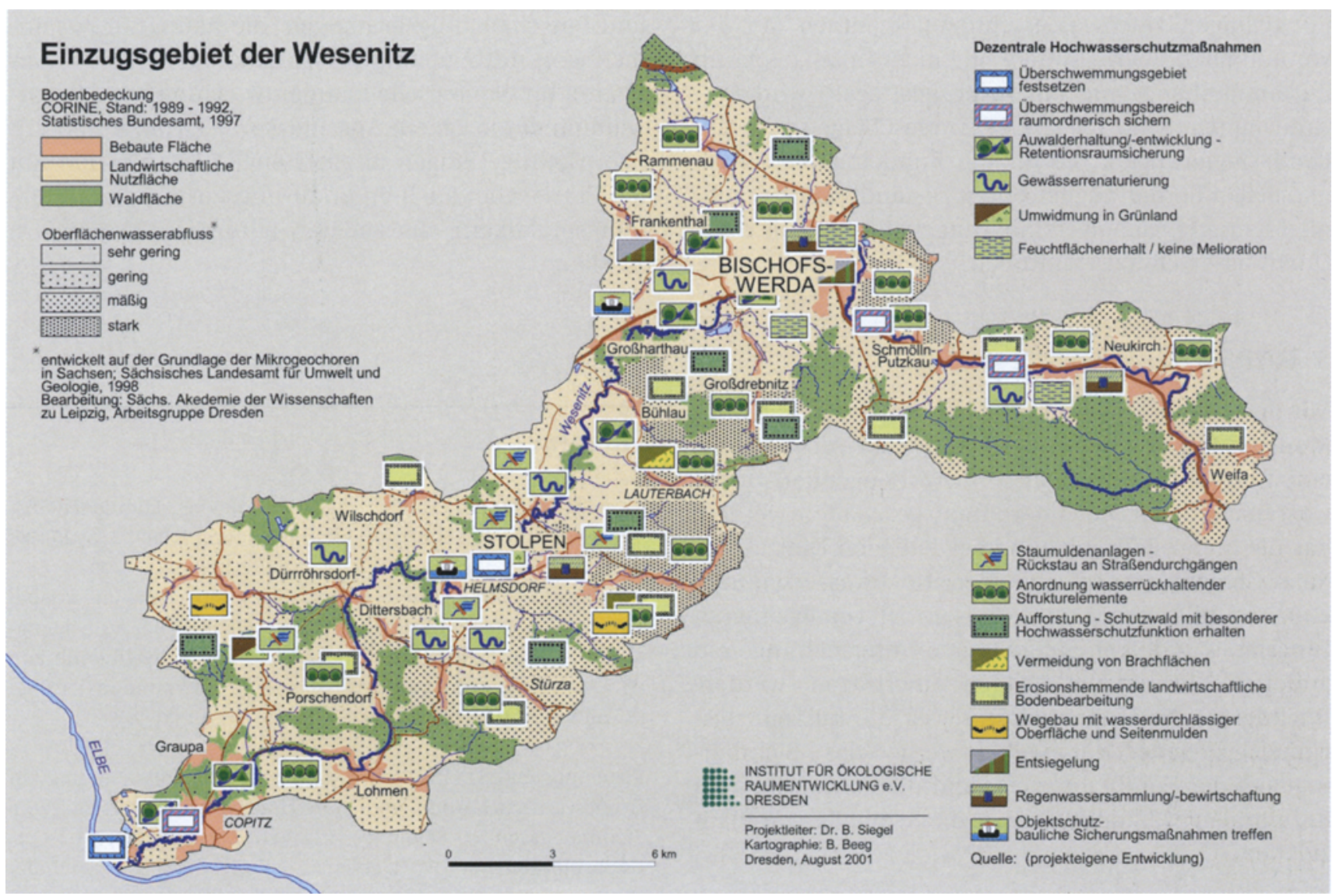

Der „Informelle Plan zum vorbeugenden Hochwasserschutz" hat empfehlenden Charakter und zielt darauf ab, sowohl Kommunen als auch Fachplanern Vorgaben zur mittel- bis langfristigen Umsetzung von Maßnahmen zum vorbeugenden Hochwasserschutz zu übermitteln.

Bei der Gestaltung des Plans ist auf eine hohe Transparenz der Planaussagen zu achten, damit die Maßnahmen nachvollzogen und die Umsetzung kontrolliert werden können. Der Plan sollte in den Kommunen der Öffentlichkeit zugänglich gemacht werden, um das Hochwasserbewusstsein in der Bevölkerung $\mathrm{zu}$ stärken. Jeder Einzelne soll durch den Plan Impulse erhalten, künftig noch mehr präventiv für den Hochwasserschutz auf dem eigenen Flur- bzw. Grundstück tätig zu werden. Auf diese Weise erzeugte Synergieeffekte sind für das Allgemeinwohl von hohem Wert und kosten den Staat nichts. Außerdem entwickelt sich aus der flusseinzugsgebietsbezogenen Planübersicht ein Zusammengehörigkeitsgefühl und ein „Miteinander-Planen“ zwischen Ober- und Unterliegergemeinden. Durch eine flusseinzugsgebietsbezogene Plandarstellung erscheint es auch eher überzeugend, erforderlich werdende Investitionen für den vorbeugenden Hochwasserschutz im Sinne einer übergeordneten Flussgebietsgemeinschaft auf die im Einzugsbereich liegenden Gemeinden anteilmäßig zu verteilen.

Da im Prinzip alle Maßnahmen, die dem vorbeugenden Hochwasserschutz dienen, zu einer ökologischen Aufwertung der Landschaft einschließlich der Siedlungsbereiche beitragen, müssen zur stringenten Umsetzung dieser Hochwasserschutzmaßnahmen auch Fördermittel aus geeigneten Programmen für den Natur- und Landschaftsschutz sowie für die Landwirtschaft, z. B. im Rahmen der agrarstrukturellen Entwicklungsplanung, erschlossen werden. Vor allem die zuständigen Ministerien sollten mit Blick auf den vorbeugenden Hochwasserschutz die Bewilligung ihrer bisher ressortbezogenen Förderbeträge zur Entwicklung von Natur und Landschaft stärker mit den Zielen 
des vorbeugenden Hochwasserschutzes verknüpfen. Nur aus kommunalen Fonds lassen sich die dringend notwendigen Hochwasserschutzmaßnahmen auf der kommunalen Ebene mittelfristig nicht finanzieren. Im Zusammenhang mit Finanzierungsfragen wird von Greiving (Greiving 2000, S. 49) vorgeschlagen, mit den Landesregierungen, die für die Bündelungen von fînanziellen Fördermöglichkeiten zuständig sind, ergebnisorientierte Globalvereinbarungen über die Ziele des Hochwasserschutzes zu treffen.

\section{Fazit}

Mit dem aus einer raumordnerischen Gesamtsicht im Modellgebiet der Wesenitz entwickelten Vorschlag für einen eigenständigen Plan zum vorbeugenden Hochwasserschutz soll ein Instrument geschaffen werden, das die in der Raumplanung bestehenden Defizite auf dem Gebiet des vorbeugenden Hochwasserschutzes abbauen hilft. Der Konzeptansatz ist verallgemeinerungsfähig und kann auf adäquate Untersuchungen in anderen Flusseinzugsgebieten übertragen werden. Wichtig für die Planentwicklung ist die flusseinzugsgebietsbezogene Herangehensweise, das Sichtbarmachen der Konfliktbereiche und die Vermittlung der dezentralen Maßnahmen über die Symbolik von Planzeichen.

Für die Planungspraxis sind noch eine Reihe von Fragen zu klären, beispielsweise zur Einordnung des Planes. Der Plan könnte sowohl Bestandteil der wasserwirtschaftlichen Rahmen- bzw. Entwicklungsplanung sein, aber auch seine Erarbeitung im Rahmen der Regional- bzw. Landschaftsrahmenplanung ist denkbar. Die Darstellungsgenauigkeit verlangt für den informellen Plan allerdings eine Maßstabsebene, die zwischen Landes- und Flächennutzungsplanung bzw. wasserwirtschaftlicher Rahmen- und Fachplanung liegt. Das spricht für eine eigenständige flusseinzugsbezogene Hochwasserschutzplanung. Die schnelle Bearbeitung favorisiert die Einordnung in das System der Raumplanung. Zu klären wäre ferner, ob vor dem Hintergrund der europäischen Bemühungen zur Einführung flussgebietsbezogener Strukturen auch entsprechende Hochwasserzweckverbände oder andere Flussgebietskörperschaften gebildet werden sollten, die die Umset- zung der Maßnahmen auslösen, überwachen und die Finanzierung steuern, auch im Hinblick auf Aufwandsund Entschädigungsleistungen, die häufig im Zusammenhang mit Nutzungseinschränkungen stehen. Das Institut für ökologische Raumentwicklung hat sich vorgenommen, in einem Anschlussprojekt diese und andere wichtige Fragen in einer engen Kooperation mit der Praxis, aber auch im Erfahrungsaustausch mit Planungspraktikern aus anderen europäischen Ländern zu klären.

\section{Literatur}

Greiving, S. (1999): Hochwasserschutz in der räumlichen Planung. In: Raumforschung und Raumordnung (1999) 1, S. 25-34

Greiving, S. (2001): Raumordnung, Regionalplanung und kooperative Regionalentwicklung und ihre Aufgaben beim Risikomanagement der Naturgefahr Hochwasser. In: Umweltbundesamt (Hrsg.): Vorbeugender Hochwasserschutz auf kommunaler Ebene. Berlin. = UBA-Texte 14 (2001), S. 39-51

Kistenmacher, H. (1995): Der Beitrag der Regionalplanung zur Grundwassersicherung und zum Hochwasserschutz. In: Vom Stadtbauwesen zur Siedlungswirtschaft, Kaiserslautern, 1.1-1.9. = Schriftenreihe des Fachgebietes Siedlungswasserwirtschaft der Universität Kaiserslautern, 7 (1995), 1.6

MKRO - Ministerkonferenz für Raumordnung (2000): Handlungsempfehlungen zum vorbeugenden Hochwasserschutz. In: GMBI, 27 (2000), S. 514-523

Pessel, S. (1997): Landschaftsökologischer Hochwasserschutz. Kassel. = Arbeitsbericht A 125 des Fachbereichs Stadtplanung/ Landschaftsplanung der UniGh Kassel, S. 25

Dipl.-Ing. Gerhard Richter

Dr.-Ing. Bernd Siegel

Institut für ökologische

Raumentwicklung e.V., Dresden

Weberplatz 1

01217 Dresden

E-Mail: gerhard.richter@ioer.de

E-Mail: b.siegel@ioer.de 